\begin{tabular}{|c|c|}
\hline \multirow{3}{*}{ 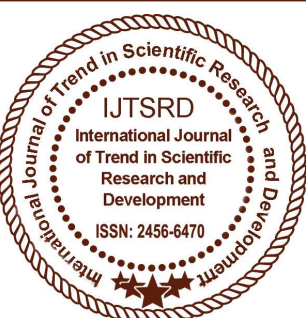 } & $\begin{array}{l}\text { International Journal of Trend in Scientific } \\
\text { Research and Development (IJTSRD) }\end{array}$ \\
\hline & International Open Access Journal \\
\hline & ISSN No: 2456 - 6470 | www.ijtsrd.com | Volume - 2 | Issue -3 \\
\hline
\end{tabular}

\title{
Image Processing Application for Vehicle Seat Vacancy Identification
}

\author{
Nikita A. Rekhate, Dr. V. S. Gulhane \\ Department of Information Technology, \\ Sipna College of Engineering and Technology, Amravati, India
}

\begin{abstract}
\end{abstract}
The importance of embedded applications on image and video processing domain has been taking a larger space. The image processing is the study of several algorithms that takes an image as input and returns an image as output. Specially we would like to elaborate our experience on the significance of computer vision as one of the domains where hardware implemented algorithms executes far better than those implemented from end to end software. Face detection is becoming one of the maximum interesting topics in the computer vision literature. The survey is directed to examine the face detection techniques. Image processing techniques is very widely held at presentday for face detection and gender detection. In this study, face detection technique is used for detecting and counting the number of passengers in electric vehicle over webcam. The webcam is installed in electric vehicle and attached with Raspberry Pi 2 model $\mathrm{B}$. When electric vehicle run off from the station, webcam will capture passenger's images in the seating area. The images will be adjusted and enhanced to decrease the noise which is concluded by software application. The images are sent in the direction of the server by $3 \mathrm{G}$ communication. Then and there, the server progression the images by means of face detection technology and counting the number of passengers in electric vehicle. The system gets the supreme amount of passengers in electric vehicle that procedure over the images then evaluates the seat vacancy of the electric vehicle.

Keywords: Face detection, Haar-like features, Morphological image processing, Contrast limited adaptive histogram equalization.

\section{Introduction}

Presently, supreme people usage of public vehicle on behalf of separate car by means of a result of the increasing of gasoline rates and traffic jams. Public company has been refining the system for display the place of the passenger vehicle for appropriateness of customers. But, individuals systems first show the place of the vehicle but not display the availability of seats in the vehicle. Customers will spoil a period for waiting the next passenger vehicle and cannot accomplish the time travel otherwise activities properly. If customers be familiar with both of the location of the passenger vehicle in addition to vacancy of seats, customers can use the period to further events earlier the passenger vehicle reaches. Customers can plan their travel well. In this study, the seat vacancy identification system is designed by means of image processing technique. Webcam is associated with Raspberry Pi 2 in the electric vehicle for detecting the object on vehicle. Webcam capture image specified image is resize then convert that RGB image to gray scale image. Later face detection is used. This face detection differentiates faces from non-faces. God has given every person a matchless face. Face is the most important part of our body, hence that it can replicate many emotions of a person. There are traditional ways are being utilized even today but with vast resources wanted to be more secured. After face detection HOG feature extraction is done. This system use Open Source Computer Vision (Open CV) to analyze and process the data then calculated the vacancy of the electric vehicle by using the maximum face detection data. In this novel approach, we are using face detection \& gender detection system. 


\section{Literature Review}

"Real-Time Integrated CCTV Using Face and Pedestrian Detection Image Processing Algorithm for Automatic Traffic Light Transitions", this exploration lessons the traffic light for pedestrian that wishes to cross the street. If the pedestrian cross the street they press the button and wait for traffic light. This system usage CCTV as an alternative the button and usage image processing for noticing the face of pedestrian. If CCTV spots the face of pedestrian, the system will fixed the red light to display for 45 second. Conversely if CCTV does not spot the face, the red light will display for only 30 second [1]

"Analyzing Impact of Image Scaling Algorithms on Viola - Jones Face Detection Framework", this exploration studies the Viola-Jones algorithm about the problem from low quality of the image and discovery the improve solution from Viola-Jones algorithm. The system usages two approaches to scaled image that are window scaling and image scaling. The image scaling has 5 techniques that is Nearest Neighbour, Bi-Linear, Bi-Cubic, Extended Linear, and Piece-Wise Extended Linear. The system usages 5 difference face database for relating the presentation of 5 different image scaling techniques. The system was established by using $\mathrm{C}++$, Visual studio 2010, and Open Source Computer Vision (OpenCV). They used confusion matrix that compose of True Positive, False Positive, and False Negative to calculate the performance of each technique. From the result, they found that the analysis in set-up of the window scaling is improved than image scaling.

[2] "FACE DETECTION USING COMBINATION OF SKIN COLOR PIXEL DETECTION AND VIOLA-JONES FACE DETECTOR", this examination studies the detection of the human skin. It usages a grouping of two techniques that are a novel hybrid color models and Viola - Jones algorithms. Its determination is to recognize the object is human or not. The system is planned in MATLAB and usage ECU face and skin database to calculate the correctness. From the result, this method has great performance more than another. When usage this method with Viola - Jones face detector, it will be more capable must be indented.

In 2014, Myunghoon [3] used Active shape model (ASM) to remove 77 facial points. Active Shape Model is widespread geometric based style in which detected image is iteratively deformed to fit shape model and extract facial points later contrast with shape model.

In 2014, Kamlesh [4] used combination of Active Appearance Model (AAM) and Local Binary Pattern (LBP) as an example of hybrid attitude, removed 68 facial points, in which AAM is geometric based method and LBP is presence based method. Subsequently extractions of features, unlike classifiers are used for the classification of emotions.

Maryam Moghaddam and Aped Meshiqini presented automatic facial recognition for used the local directional pattern to signify the geometry and examine the performances template matching and support vector machine are used and enhanced algorithm entropy LDP+SVM [5].

Dalal et al. [6] found the Histogram of Oriented Flow (HOF) features (describing motion) by means of optical flow images. However, the marks found by means of motion features presented simply little enhancement over its counter.

\section{Proposed Work}

The goal of our dissertation is to make available identification vacant seat system by means of face detection and gender detection on raspberry pi board and display the entire outcome in web panel. It shows how many seat are vacant or allocated and gender of that specific person is also displayed in web panel. This will usage to saving time of person for waiting hour and hour of vehicle. People manage their time in other activities until vehicle reach. People can plan their travel better.

Actual work is completed when the electric vehicle leaves from the station, the system will capture the image in the passenger seat area (1 image per 1 second). The images will be adjusted and enhanced to diminish the noise which is completed by software application. The capture images are transferring to the raspberry pi kit for further processing. Web panel is used to display result of our system. 


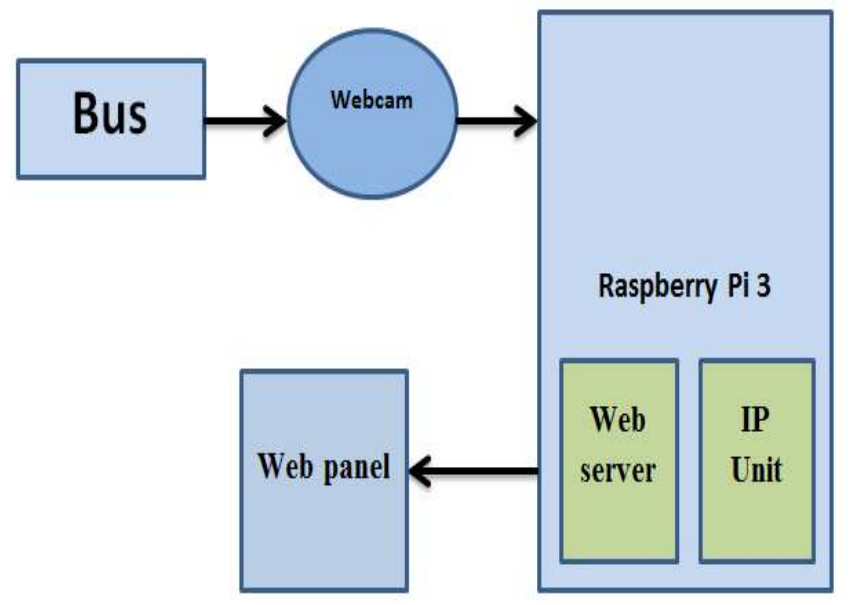

Fig.1: System Architecture

\section{Implementation}

Below figure shows that the flowchart of implementation of human face detection and gender detection system using raspberry pi.

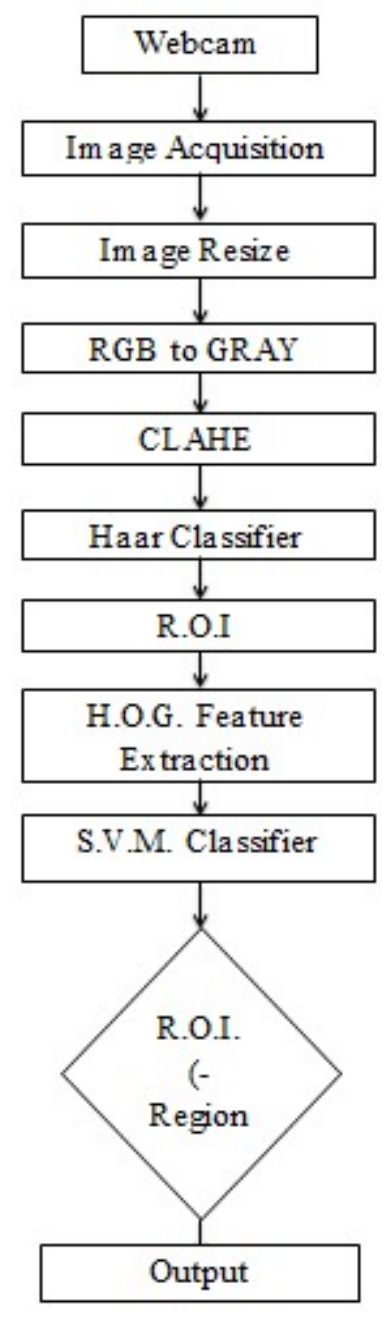

Fig.2: Flowchart

\section{Step 1: Setting up Raspberry PI}

SD card into the SD card slot on the Raspberry Pi, which will only fit one way. Next, plug the USB keyboard and USB mouse into the USB slots on the Raspberry Pi. Make assured that the monitor or TV is turned on, and that the right input is selected. Then Connect the HDMI cable from your Raspberry Pi to the monitor or TV. If the Raspberry Pi is needed to be connected to the internet, plug an Ethernet cable into the Ethernet port next to the USB ports. When all the required cables and SD card are plugged then, plug in the micro USB power supply. First of all the Linux OS is installed into the Raspberry Pi board via Micro SD card and appropriate code is written in Python language for the object detection using the Open CV libraries and is dumped in the board. The USB Camera is interfaced; the GPIO pins are programmed using commands in Linux and Python in this stage. The camera is interfaced to the Pi via the USB port and the door lock module is interfaced via the GPIO pins on the Pi.

\section{Step 2: Image Acquisition}

Digital image acquisition is the creation of photographic images, such as of a physical scene or of the interior structure of an object. The term is often assumed to imply or include the processing, compression, storage, printing, and display of such images. Webcam connect to the raspberry pi kit. When person standing in front of camera then webcam capture face image of that person. In image Acquisition When images are read it is divided into rows and columns means row wise features column wise pixels.

\section{Preprocessing}

\section{Image Resize \\ 2. RGB to Gray Scale \\ 3. CLAHE}

The data which is collected from Input unit that is captured Image and Video frames input is fed into the processing unit. Here the processing unit is nothing but a Raspberry Pi board. Along with code scripts of the implemented modules. Resize the captured face image into $400 \times 400$. Given image is in the form of 8 bit color image. Each pixel encompass three data for red, green, blue components(RGB) and each component range from 0 to 255.RGB image contains lots of data which may not be required for further processing. So convert RGB image to grey scale 
image. Then apply CLAHE method. Contrast limited adaptive histogram equalization or CLAHE is the process for increasing the image quality. This method considers the data of histogram equalization in each of pixel of grey scale format. In the first step, this method finds average histogram value of the image. The method uses the histogram value that has higher than the average value to share to all pixels inside the image for equal histogram value.

\section{Step 3: Face detection}

In face detection process haar cascade algorithms are used for face detection.

- First capture face image by using webcam then resize the image.

- Haar-like features are digital image features used in object detection.

- Initially, the algorithm needs a lot of positive images (images of faces) and negative images (images without faces) to train the classifier.

Then we need to extract features from it. In this face detection process, captured colour face image is converted into black and white image i.e. grey scale image. It reduces the number of pixel for simplification of detection. Then selection of ROI take place i.e. face detection process is done.

\section{Step 4: Feature Extraction}

In feature extraction process HOG the most successful stand-alone descriptor for supervised classification. The main idea behind the histogram of oriented gradient is that the local appearance and shape of object in an image can be described by the intensity distribution of gradients or direction of the contours.

The implementation of these descriptors can be obtained by

- Dividing the image into small connected regions, called cells.

- Each cell we compute a histogram of gradient directions or edge orientations for all pixels of the cell. The combination of these histograms is the descriptor.

- Create 9 sub-images called binaries images, as we have chosen the size of the histogram box equal to $20^{\circ}\left(180^{\circ} / 20^{\circ}=9\right)$. All pixels in these images are set to zero except the pixels in the original image for which the values of the gradient orientation correspond to the particular case. For each cell of size $8 \times 8$ pixels, we compute the values of the 9 boxes of gradient orientation histogram.

- For a block of $2 \times 2$ cells, we calculate the HOG descriptor for each cell, then the obtained arrays are assembled into a single array of 36 components.

\section{Step 5: SVM Classifier}

The set of descriptor is used to feed the SVM classifier, which generates a model (a set of support arrays). During the decision phase, the descriptors are calculated in an identical manner as in the learning phase. Decision making, regarding the class membership is made directly by the decision function of SVM. In our experiments, we use the linear kernel function to classify the descriptors given by the learning database.

\section{Step 6:Output}

Overall output will be seen in webpage. In place of URL type IP address of raspberry pi kit. Then we get $\log$ in page. After log in we get output.

\section{Experimental Result}

The experiment use dissimilar number of passengers and experimental time. Three experiments are conducted. The passengers in electric vehicle are not equivalent in every round. In each experiment usages unlike number of images to calculate the correctness of face detection in electric vehicle.

From the result, the number of images has an effect for face detection. If we usage less images, the program will be little performance and correctness. The program cannot identify the face for the reason that the face of passengers is not pure. This problem consists from environment around the vehicle. It makes the images too light or dark. If the number of image is rises (long capturing time), the movement of passenger face is rise as well. The program can identify the face of the passenger enhanced because the program has a additional chance to spot the passengers face from numerous images.

\section{A. Graphical Analysis:}

In analysis, we show detection rate of face. $\mathrm{X}$ axis contain false positive. $\mathrm{Y}$ axis contain detection rate. Detection with preprocessing is more accurate than without preprocessing. 


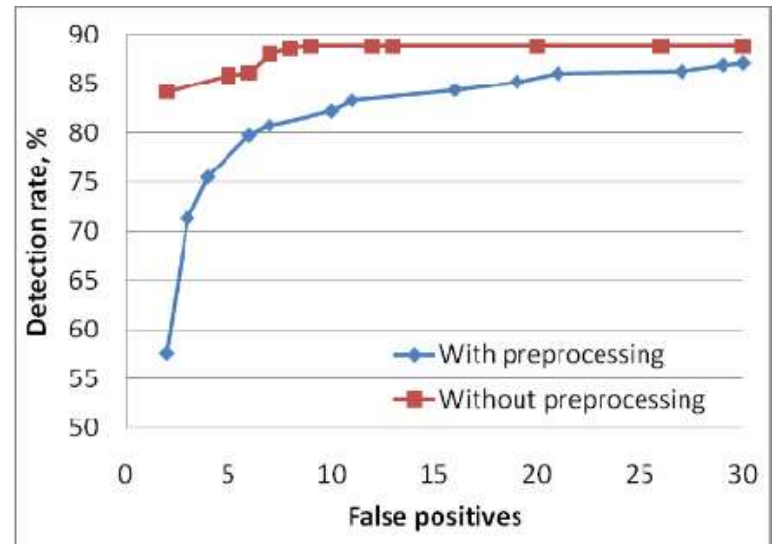

Fig.3: Face Detection Analysis

4) Kamlesh Mistry, Li Zhang, Siew Chin Neoh, Ming Jiang, Alamgir Hossain, Benoît Lafon, "Intelligent Appearance and Shape based Facial Emotion Recognition for a Humanoid Robot" Software, Knowledge, Information Management and Applications (SKIMA), 2014 8th International Conference, pp 18, Dhaka.

5) Unsoojang, Eui Chul lee,'’Performance Analysis of Pixel Based Face Recognition Method, 'IJBSBT Vol.8, No. 2(2016), PP.197-206.

6) Dalal N. and Triggs B. 2005. Histograms of Oriented Gradients for Human Detection. In Proceedings of IEEE International Conference on Computer Vision and Pattern Recognition.

\section{CONCLUSION}

This system proposed a system for face detection and gender estimation technique. Also some popular wellknown face detection technique is described. Face detection technique have been employed in different applications such as face recognition, facial feature extraction. Webcam and Raspberry Pi were installed in electric vehicle. When the electric vehicle leave from the station, webcam captured the images and send to the server by using Raspberry Pi/and $3 \mathrm{G}$ communications. The images were sent completely. The number of images has a direct impact to the face detection result. If the number of images increases, the accuracy of face detection is increase as well. Because the system will have more chance to detect the passenger face from many images.

\section{References}

\section{ISSN: $2456-6470$}

1) Cyrel O.Manlises, Jesus M. Martinez Jr., Jackson L.Belenzo, Czarleine K. Perez, and Maria Khristina Theresa A. Postrero, "Real-Time Integrated CCTV Using Face and Pedestrian Detection Image Processing Algorithm For Automatic Traffic Light Transitions", School of Electrical, Electronics and computer Enfineering, Mapua Institute of Technology, 2015

2) GUAN-CHUN LUH, "FACE DETECTION USING COMBINATION OF SKIN COLOR PIXEL DETECTION AND VIOLA-JONES FACE DETECTOR", Deprtment of Mechanical Engineering, Tatung University, 2014

3) Myunghoon Suk, Balakrishnan Prabhakaran, Real-time Mobile Facial Expression Recognition System - A Case Study, Computer Vision and Patter Recognition Workshops (CVPRW), IEEE Conference, pp 132 - 137, 2014. 